\title{
Patrones de uso de fauna silvestre en el bajo río San Jorge, Sucre, Colombia
}

\author{
Patterns of use of wildlife in the low San Jorge River, Sucre, Colombia
}

VALENCIA-PARRA, EFREN” Esp, DE LA OSSA V, JAIME² Ph.D.

${ }^{1}$ Universidad de Sucre - SUE Caribe, Colombia. ${ }^{2}$ Universidad de Sucre, Facultad de Ciencias Agropecuarias, Grupo de Investigación en Biodiveridad Tropical, Colombia.

\section{Keywords:}

Wildlife; use; Mojana; Sucre; Colombia.

\section{Palabras Clave:}

Fauna silvestre; uso; Mojana; Sucre; Colombia.

\section{Abstract}

The present work collects information obtained by surveys on the use patterns and vulnerability of wildlife for an area of the ecoregion of the Mojana belonging to the low San Jorge River. It evidences as decreases this ecosystemic component due to the strong antropic pressure that suffers and discusses the social causes that impact in this negative phenomenon with more prevalence. It is the first register of the massive motorcycles use for the wildlife traffic, and is determinate a high extractive dependence with participation of all the age categories, with marked masculine linking.

\section{Resumen}

El presente trabajo recopila información obtenida mediante encuestas sobre los patrones de uso, vulnerabilidad de fauna silvestre para la zona de la ecoregión de la Mojana perteneciente al bajo río San Jorge. Evidencia como existe una disminución de este componente ecosistémico debido a la fuerte presión antrópica que sufre y discute las causas sociales que con mayor prevalencia inciden en este fenómeno negativo. Se registra por primera vez el uso masivo de motocicletas para el tráfico de fauna silvestre y se determina una alta dependencia extractivista con participación de todos los grupos de edad, con marcada vinculación masculina. 


\section{Introducción}

En cuanto a la fauna silvestre, en la región de La Mojana se han realizado varios estudios que han pretendido caracterizar sus patrones de uso y comercialización a partir del desarrollo de encuestas o entrevistas semiestructuradas principalmente a cazadores, extractores de fauna y comerciantes, con el fin de establecer el volumen, la frecuencia de consumo, relacionar los factores sociales y familiares que se presentan en el proceso de comercialización, así como reconocer los aspectos comerciales más importantes que se generan en el proceso de aprovechamiento faunístico; en este sentido, se podría decir que los trabajos realizados por AGUILERA y NEIRA (1999), AGUILERA-DÍAZ (2004), son pioneros en el estudio de los patrones anteriormente descritos, pues sirvieron de punto de partida para numerosos estudios posteriores, como el de DE LA OSSA (2003), en el que se hace un diagnóstico de los aspectos más importantes relacionados con los usos de la fauna silvestre en la región de La Mojana y que analiza también las posibilidades de manejo que se podrían implementar para conciliar la oferta ambiental con la demanda social, con el fin de consolidar programas investigativos y productivos de desarrollo sostenible, que sean pertinentes y busquen una solución de tipo global.

La sobreutilización de la fauna silvestre y el peligro de extinción que enfrenta, en algunas zonas, se debe principalmente a una problemática socioeconómica muy compleja y precaria donde los pobladores no tienen acceso a trabajos bien remunerados debido a la falta de estudios secundarios, universitarios y el analfabetismo de muchos, situación que los obliga a buscar su sustento diario e ingresos adicionales que les ayudan con gastos en su cotidianidad mediante la explotación de los recursos naturales (fauna), mediante la caza de subsistencia, los cuales son utilizados principalmente para carne de consumo o venta, tenencia de mascotas, consumo o venta de huevos, venta de crías, venta de piel, medicinal y caza por daños en sembrados o producción. En algunos casos como en el comercio de la Hicotea es realizado a través de intermediarios, los cuales compran en grandes cantidades; y para llenar este mercado se utilizan técnicas de captura muy perjudiciales al ecosistema como son las quemas (SAMPEDRO et al., 2003; DE LA OSSA et al., 2011).

En el presente estudio se Determinan los patrones de uso, vulnerabilidad y estrategias de conservación para la fauna silvestre utilizada en la subregión del bajo río San Jorge, Caribe colombiano.

\section{Materiales y métodos}

Área de estudio: La subregión del bajo río San Jorge, perteneciente a la depresión momposina, es un sistema ambiental que actúa como regulador de los ríos Magdalena, Cauca y San Jorge; mayoritariamente la zona está conformada por humedales (AGUILERA-DÍAZ, 2004); además de poseer importantes variados hábitats para la fauna silvestre regional y ser por excelencia un lugar de tradicional extractivismo (DE LA OSSA, 2003). En su gran mayoría la zona está conformada por llanuras fluvio deltaicas y fluvio lacustres; con zonas de bosque húmedo tropical, bosque seco tropical, bosque muy seco tropical y sabanas naturales (AGUILERA-DÍAZ, 2005). La Subregión San Jorge está localizada en la parte suroccidental del departamento, compuesta por los municipios de San Marcos, San Benito Abad, La Unión y Caimito, los cuales tienen un territorio de $2.934 \mathrm{Km}^{2}$, representa el $28,3 \%$ del total del departamento de Sucre (DANE, 2012) (Figura 1).

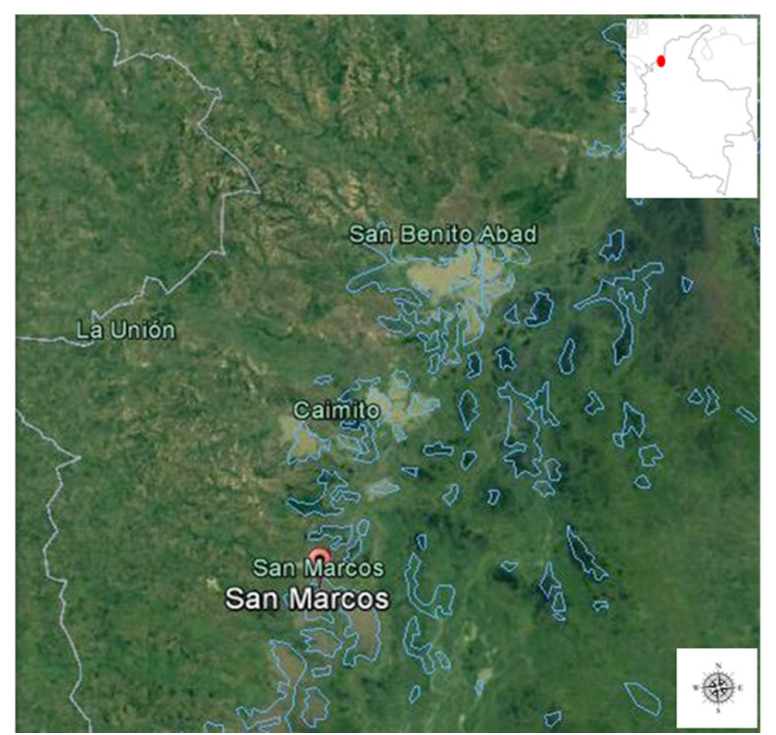

Figura 1. Zona de estudio (Google earth versión libre) 
La zona pertenece bioma tropical alternohígrico y hace parte del cinturón árido pericaribeño (HERNÁNDEZ-CAMACHO y SÁNCHEZPÁEZ, 1992). El Clima se clasifica típicamente Tropical, con influencia de los vientos Alisios que predominan durante algunos meses del año, y de la situación en la Zona de Confluencia Intertropical (ZCIT), los cuales determinan variaciones en humedad relativa y precipitaciones, determinando las condiciones climáticas prevalecientes a lo largo del año. Con una precipitación anual entre 900 y $1.200 \mathrm{~mm}$, con temperatura media anual de $25^{\circ} \mathrm{C}$ y humedad relativa media del $80 \%$. Se presentan dos estaciones marcadas: una seca con duración aproximada de Cinco (5) meses (diciembreabril), y otra estación lluviosa (Finales de abril Finales de noviembre) con algunas disminuciones sustanciales entre junio y agosto, denominado "veranillo de San Juan" (CARSUCRE, 2012).

Colecta de información: Para la identificación de los usos de la fauna se realizaron entre agosto del 2014 y enero del 2015 un total de 12 sesiones de trabajo en los puertos fluviales de San Marcos, Caimito y San Benito Abad en el departamento de Sucre; Según DE LA OSSA y DE LA OSSA-LACAYO (2011) se aplicaron un total de 200 encuestas semiestructuradas a pescadores $y / 0$ extractivistas previamente identificados, procedentes de la zona inundable y con permanencia en la zona de por lo menos 10 años. Adicionalmente se llevó a cabo una comprobación de imágenes para reconocimiento de cada especie registrada en las encuestas.

Análisis de información: Los datos fueron organizados en tablas de acuerdo con la respuestas obtenidas y se le aplicó estadística no paramétrica con cálculo de porcentaje, media y desviación estándar.

\section{Resultados y discusión}

En cuanto tiene que ver con la edad de las personas encuestadas el $60 \%$ corresponde a adultos mayores de 60 años, seguido de edades que oscilan entre los 40 a 59 con un $35 \%$ y solo $5 \%$ corresponde a personas entre los 20 a 39 años de edad; estos resultados permiten observar que las personas dedicadas a tareas relacionadas con el diario trasegar en los puertos son de avanzada edad. En relación al sexo de los encuestados se tiene que la mayoría de las personas son de sexo masculino con un $75 \%$ y el restante $35 \%$ son de sexo femenino.

El nivel de escolaridad demuestra que un $60 \%$ de la población encuestada no posee ningún nivel de educación escolarizada, el $20 \%$ cuenta con certificación de básica primaria y el $10 \%$ con básica secundaria; otro $10 \%$ afirmó haber realizado algunos cursos complementarios relacionados con diferentes actividades informática básica.

El $30 \%$ afirmó dedicarse a oficios varios como trabajador independiente en labores como vendedores ocasionales, jornaleros, cargue y descargue de mercancías en los puertos, ayudantes de construcción, entre otros. Como agricultores solo el $18 \%$ afirmó dedicarse a esta labor utilizando los predios donde habitan, están asentados y/o parcelados. Solo el $2 \%$ está empleado como impulsador de algún producto de tipo comercial, ventas en algún local comercial o servicios generales. El 50\% afirmó dedicarse al mototaxismo y que en temporadas de aprovechamiento de fauna silvestre aprovechan para vincularse al tráfico ilegal de productos de fauna silvestre (Figura 2).

La información social antes referida coincide con los señalado por DE LA OSSA (2003), DE LA OSSA et al. (2011), DE LA OSSA y DE LA OSSALACAYO (2011), DE LA OSSA-LACAYO y DE LA OSSA (2012a y 20012b). No obstante, por primera vez se detecta que el uso de motocicletas como una estrategia de transporte, cuya versatilidad y los tipos de vías por la cuales se desplazan las hacen de difícil control para las autoridades ambientales; sin duda se trata una modalidad de transporte que dificulta el control del tráfico y ayuda a incrementar la vulnerabilidad de la fauna silvestre ya que los controles se tornan poco eficientes en tal sentido.

La finalidad de la cacería demuestra en un $60 \%$ que las personas comercializan los productos de la cacería para la obtención de recursos para inversión en el núcleo familiar, representados en: alimentos, arriendos y estudio entre otros. Solo el $26 \%$ lo utilizan para consumo como base de proteína de origen animal, para usos medicinales el $5 \%$ menciono su utilización, otro $5 \%$ para controlar animales plagas de sus sistemas de 

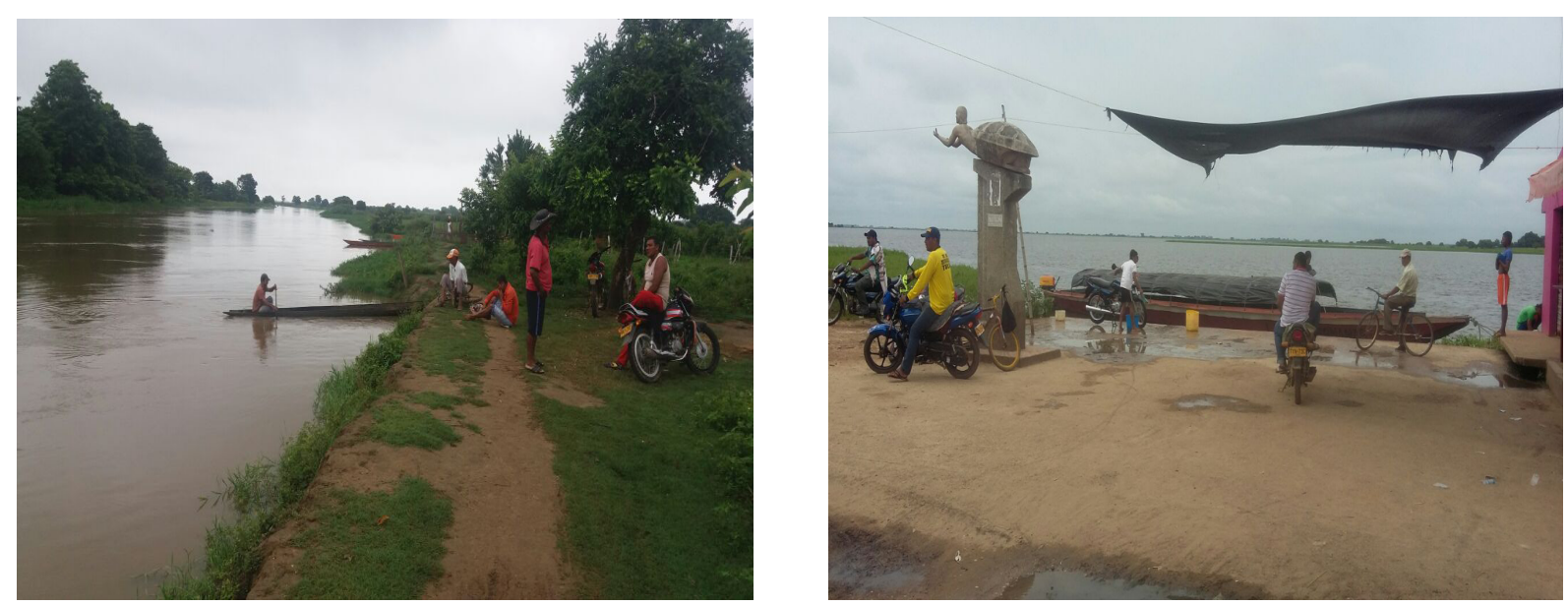

Figura 2. Presencia de motos en las zonas portuarias de la zona de estudio.

producción agropecuaria, situación que coincide con los determinado por DE LA OSSA y DE LA OSSA-LACAYO (2011) y con DE LA OSSALACAYO y DE LA OSSA (2012a).

Para las faenas de caza se constató que el $40 \%$ de los animales aprovechados son hicotea (Trachemys callirostris), seguido de pisingo (Dendrocyna autumnalis) en un $20 \%$, babilla (Caiman crocodilus fuscus), ponche (Hydrochaeris hydrochaeris) e Iguana (Iguana iguana) con un $10 \%$ y un $5 \%$ para otras especies de fauna silvestre, lo cual está de acuerdo a lo planteado por DE LA OSSA-LACAYO y DE LA OSSA (2012b). En general las especie utilizadas que se detectan en este estudio corresponden a las que de manera general son objeto de cacería para América tropical (PÉREZ y OJASTI, 1996; RAMÍREZ-PERILLA, 1996; POLANCO, 2000; AGUILERA-DÍAZ, 2004) y de forma específica para la zona de estudio (DE LA OSSA-LACAYO Y DE LA OSSA, 2012a, 2012b) (Tabla 1).

En relación a la preferencia por el sexo de los animales cazados nos muestra que un $60 \%$ son hembras debido al consumo de huevos de algunos quelonios, un $30 \%$ prefiere cazar machos y el $10 \%$ no tiene en cuenta el sexo de los animales al momento de la faena. El $60 \%$ de los encuestados mencionan preferencia por cazar animales adultos, un 30\% mencionan cazar animales jóvenes y el $10 \%$ de los encuestados no tiene preferencia por el estado de desarrollo de los animales cazados.

Los datos respecto de estadio de desarrollo de los ejemplares cazados son según OJASTI (2000) producto de los intereses del mercado, que es el sistema que direcciona la mecánica de las capturas; no obstante es importante reconocer, como lo plantean REDFORD y ROBINSON (1991), la selectividad podría estar más relacionada con la venta pero no es un factor que frene su uso general pues el autoconsumo juega un importante papel. Igualmente es necesario reconocer que en muchas regiones del mundo, la cacería local con fines de subsistencia es la única alternativa para la obtención de proteína de origen animal (ALVARD, 2000; HILL y PADWE, 2000).

\section{Conclusión}

Los procesos de uso comercial y consumo de subsistencia de fauna silvestre de interés económico en la zona de estudio, de acuerdo con los estudios previos citados y los resultados de este trabajo, se muestran como un proceso continuo que no ha dejado de existir en función del tiempo. Se denota una alta influencia cultural y por primera vez se evidencia el uso de motos para el tráfico ilegal de productos faunísticos, fundamentado en la versatilidad que para su movilidad estos vehículos poseen. 
Tabla 1. Patrones de uso para reptiles, aves y mamíferos en la zona de estudio.

\begin{tabular}{|c|c|c|c|c|c|c|c|c|c|c|}
\hline Especie & N. Común & Tax. & $\mathrm{C} / \mathrm{C} / \mathrm{V}$ & $\mathrm{M} / \mathrm{C}$ & $\mathrm{H} / \mathrm{C} / \mathrm{V}$ & $\mathrm{c} / \mathrm{V}$ & $\mathrm{P} / \mathrm{V}$ & M & D & Total \\
\hline Iguana iguana & Iguana & $\mathrm{R}$ & 1 & 1 & 1 & 1 & & 1 & & 5 \\
\hline Tupinambis teguixin & Lobo pollero & $\mathrm{R}$ & & & & & & 1 & & 2 \\
\hline Boa constrictor & Boa & $\mathrm{R}$ & & 2 & & & 1 & 1 & & 4 \\
\hline Trachemys callirostris & Hicotea & $\mathrm{R}$ & 1 & 2 & 1 & 2 & & 1 & & 7 \\
\hline Rhinoclemmys melanosterma & Galapago & $\mathrm{R}$ & 1 & 1 & 1 & 1 & & & & 4 \\
\hline Podocnemys lewyana & Tortuga & $\mathrm{R}$ & 1 & 1 & 1 & 1 & & & & 4 \\
\hline Caiman crocodilus fuscus & Babilla & $\mathrm{R}$ & 1 & & & 2 & 2 & 1 & & 6 \\
\hline Phalacrocorax olivaceus & Pato yuyo & A & 2 & 1 & & & & & & 3 \\
\hline Ardea cocoi & Garza morena & A & 1 & 1 & & 1 & & & & 3 \\
\hline Mycteria americana & Coyongo & A & 1 & & & 1 & & 1 & & 3 \\
\hline Chauna chavaria & Chavarri & A & 1 & 1 & & 1 & & & & 3 \\
\hline Dendrocygna viduata & Viudita & A & 2 & 1 & & 1 & & 1 & & 5 \\
\hline Dendrocygna autumnalis & Pisingo & A & 2 & & & 1 & & 1 & & 4 \\
\hline Cairina moschata & Pato real & A & 1 & 1 & & 1 & & 1 & & 4 \\
\hline Pandion haliaetus & Aguila pescadora & A & & 1 & & & & & & 1 \\
\hline Buteo magnirostris & Gavilan pollero & A & & 1 & & 1 & & & & 2 \\
\hline Milvago chimachima & Pigua & A & & 1 & & & & & & 1 \\
\hline Ortalis garrula & Guacharaca & A & & 1 & & 1 & & & & 2 \\
\hline Penelope purpurascens & Pavita & A & 1 & 1 & 1 & 1 & & & & 4 \\
\hline Colinus cristatus & Perdiz & A & 1 & 1 & & 1 & & & & 3 \\
\hline Burhinus bistriatus & Galan & A & 1 & 1 & & & & & & 2 \\
\hline Columba cayennensis & Tierrerita & A & 1 & & & & & & & 1 \\
\hline Columbina talpacoti & Tortolita & A & 1 & & & & & & & 1 \\
\hline Leptotila verrenauxi & Caminera & A & 1 & & & & & & & 1 \\
\hline Ara arauna & Gonzalo & A & & 1 & & 1 & & & & 2 \\
\hline Aratinga pertinax & Cotorra & A & & 1 & & 1 & & & 1 & 3 \\
\hline Brotogeris jugularis & Perico & A & & 2 & & 1 & & & 1 & 4 \\
\hline Amazona ochrocephala & Loro & A & & 1 & & 1 & & & & 2 \\
\hline Ramphastos sulfuratus & Guazale & A & & 1 & & & & & & 1 \\
\hline Thraupis episcopus & Azulejo & A & & 1 & & & & & 1 & 2 \\
\hline Volantina jacarina & Mochuelo & A & & 1 & & & & & & 1 \\
\hline Sphorophila minuta & Meriño & A & & 1 & & & & & & 1 \\
\hline Sicalis flaveola & Canario & A & & 1 & & & & & & 1 \\
\hline Didelphis marsupialis & Zorra chucha & M & & & & & & 2 & 1 & 3 \\
\hline Dasypus novemcinctus & Armadillo & M & & 1 & & & & 1 & & 2 \\
\hline Alouatta seniculus & Mono aullador & M & & 1 & & 2 & & 1 & & 4 \\
\hline Cerdocyon thous & Zorra perro & M & & & & 1 & & 1 & 1 & 3 \\
\hline Tayassu tajacu & Zaino & M & 1 & & & 1 & & 1 & & 3 \\
\hline Mazama americana & Venado & M & 1 & & & 1 & & 1 & & 3 \\
\hline Hydrochoerus hydrochaeris & Ponche & M & 1 & & & 1 & & 1 & & 3 \\
\hline Cuniculus paca & Guatinaja & M & 1 & & & 1 & & 1 & & 3 \\
\hline Dasyprocta punctata & Ñeque & M & 1 & & & 1 & & 1 & & 3 \\
\hline Silvilagus floridanus & Conejo & M & 1 & & & 1 & & 1 & 1 & 4 \\
\hline
\end{tabular}

$\mathrm{R}=$ Reptil, $\mathrm{A}=$ Ave, $\mathrm{M}=$ Mamífero, $\mathrm{C} / \mathrm{C} / \mathrm{V}=\mathrm{Carne}$ (consumo o venta), $\mathrm{M} / \mathrm{C}=$ mascotas (tenencia en casa), $\mathrm{H} / \mathrm{C} / \mathrm{V}=$ huevos (consumo o venta), $\mathrm{C} / \mathrm{V}=\mathrm{crías}(\mathrm{venta}), \mathrm{P} / \mathrm{V}=$ piel (venta), $M=$ medicinal, $D=$ daños (Agricultura o producción pecuaria). 


\section{Referencias}

AGUILERA, G.E.; NEIRA, F. 1999. Comprobación y orientación de la Sostenibilidad en la región de La Mojana. Corpoica. Tibaitatá, Cundinamarca, Colombia.

AGUILERA-DÍAZ, M. 2004. La Mojana: riqueza natural y potencial económico. Banco de la República. Serie de documentos de trabajo sobre economía regional No. 48. Cartagena, Colombia.

AGUILERA-DÍAZ, M. 2005. La economía del departamento de Sucre: ganadería y sector público. Banco de la República. Serie de Documentos de trabajo sobre economía regional № 63 . Cartagena, Colombia.

ALVARD, M. 2000. The impact of traditional subsistence hunting and trapping populations: data from Wana horticulturalists of Upland Central Sulawesi, Indonesia. Págs. 214-230. En: Robinson, J.G.; Redford, K.H. (Eds.). Neotropical Wildlife Use and Conservation. Chicago University Press, Chicago, USA.

CARSUCRE. 2012. Plan de Acción 2012-2015. Corporación Autónoma Regional de Sucre. Sincelejo, Sucre.

DE LA OSSA, V.J.; OLIVERO-GÓMEZ, G.; RUIZ. J.G. 2011. Utilización de quelonios de interés económico en el municipio de Caimito, Sucre, Colombia. Rev. Col. cienc. Anim. 3 (1):3-14.

DE LA OSSA, V.J. 2003. Manejo de fauna silvestre tropical. Pags. 10-39. En: Programa de Desarrollo Sostenible de la Región de La Mojana. DNP, FAO, Bogotá, Colombia.

DE LA OSSA, V.J.; DE LA OSSA-LACAYO, A. 2011. Cacería de subsistencia en San Marcos, Sucre, Colombia. Rev. Col. cienc. Anim. 3 (2):213-224.

DE LA OSSA-LACAYO, A.; DE LA OSSA, V.J. 2012a. Utilización de fauna silvestre en el área rural de Caimito, Sucre, Colombia. Rev. Col. cienc. anim 4 (1):46-58.

DE LA OSSA-LACAYO, A.; DE LA OSSA, V.J. 2012b. Índice de valor de uso para fauna silvestre en la región del San Jorge, Mojana sucreña, Colombia. Rev. Colombiana cienc. Anim. 4 (2):308-319.

DANE - DEPARTAMENTO NACIONAL DE ESTADÍSTICA. 2012. Proyecciones de población 2011. Disponible en: http://www.dane.gov.co/. Consultado: 10-07-2016.

HERNÁNDEZ-CAMACHO, J.; SÁNCHEZ-PÁEZ. H. 1992. Biomas terrestres de Colombia. Págs. 153173. En: Halffter, G. (Ed.). La diversidad biológica iberoamericana I. Acta Zoológica Mexicana. México.

HILL, K.; PADWE, J. 2000. Sustainability of Aché Hunting in the Mbaracayú Reserve, Paraguay. Págs. 79-105. En: Robinson, J.G.; Bennett, E.L. (Eds.). Hunting for Sustainability in Tropical Forests. Columbia University. New York, USA.

OJASTI, J. 2000. Manejo de Fauna Silvestre Neotropical. SIMAB Series N 5. Smithsonian Institution / MAB Program. Washington, D. C.

PÉREZ, E.M.; OJASTI, J. 1996. La utilización de la fauna silvestre en la América Tropical y recomendaciones para su manejo sustentable en las sabanas. Ecotropicos 9 (2):71-82. 
POLANCO, R. 2000. Diagnóstico del uso y comercio de fauna silvestre en el Caribe, Pacífico, Andes, Amazonia y Orinoquia colombiana. Instituto de Investigación en Recursos Biológicos Alexander von Humboldt. Bogotá, Colombia.

RAMÍREZ-PERILLA, J. 1996. Tradición de uso y aprovechamiento de fauna silvestre: límites de la sostenibilidad y acciones posibles. Fundación Natura, Organización Regional Indígena Emberá WounaanOrewa-Organización de Estados Iberoamericanos para la Educación la Ciencia y la Cultura, Unidad Administrativa Especial del Sistema de Parques Naturales Nacionales del Ministerio del Medio Ambiente. Impreandes Presencia S.A. Bogotá, Colombia.

REDFORD, K. H.; ROBINSON, J.G. 1991. Subsistence and commercial uses of wildlife in Latin America. Pág. 6-23. En: Robinson, J.G.; Redford, K.H. (Eds.). Neotropical wildlife use and conservation. The University of Chicago Press. Chicago, USA.

SAMPEDRO. A.; ARDILA, M.; FUENTES, S. 2003. Datos sobre la reproducción de la jicotea (Trachemys scripta callirostris : Chelonia, Emydidae) en la subregión de la Mojana, departamento de Sucre, Colombia. Revista Biología 17 (2):120-125. 